\title{
Reabilitação estética e funcional em paciente com cárie severa da infância: relato de caso
}

Aesthethic and functional rehabilitation of a patient with severe childhood caries: case report Rehabilitación estética y funcional en un paciente con caries infantil severa: reporte de caso

\author{
Luciana Pontes Barros LOPES ${ }^{1}$ \\ Ary de Oliveira ALVES FILHO² \\ Pollyanna Oliveira MEDINA ${ }^{2}$ \\ Rachid Pinto ZACARIAS FILHO \\ Simone Assayag HANAN ${ }^{2}$
}

${ }^{1}$ Cirurgiã-Dentista, Faculdade de Odontologia, UFAM- Universidade Federal do Amazonas, 69033-760 Manaus-AM, Brasil

${ }^{2}$ Professor(a) Adjunto, Departamento de Odontopediatria, Faculdade de Odontologia, UFAM- Universidade Federal do Amazonas, 69033-760 Manaus-AM, Brasil ${ }^{3}$ Professor Adjunto, Departamento de Materiais Dentários, Faculdade de Odontologia, UEA- Universidade do Estado do Amazonas, 69065 -001 Manaus-AM, Brasil

\section{Resumo}

Introdução: A cárie severa da infância gera um impacto econômico, emocional e físico nas crianças e em suas famílias, além de determinar alterações funcionais, estéticas e comportamentais, as quais devem ser consideradas na efetivação de um tratamento odontológico eficaz. Objetivo: Este trabalho teve como objetivo realizar um relato de caso clínico de reabilitação estética e funcional de um paciente infantil, do sexo masculino, 5 anos de idade, acometido por CSI, atendido na disciplina de Clínica Odontológica Infantil da Universidade Federal do Amazonas. Caso Clínico: Ao exame clínico e radiográfico, foram observadas lesões cariosas cavitadas nos elementos dentários 51,52,61,62 e 64 , além de mancha branca ativa no 74. O tratamento proposto para este paciente incluiu orientação de hábitos de higiene bucal e dietéticos adequados, aplicações tópicas de flúor nas lesões cariosas ativas, necropulpectomia e restauração com resina composta fotopolimerizável. O dente 64, bem como nos dentes 51,52,61 e 62, severamente destruídos pela doença cárie, usando-se coroas de acetato. Conclusão: O tratamento mostrou-se bem sucedido, sendo possível sua realização em ambiente ambulatorial, permitindo restabelecer a função mastigatória e a estética do sorriso, além de promover a educação em saúde por meio da conscientização da criança/núcleo familiar acerca da importância do autocuidado na eliminação dos fatores etiológicos e no controle da doença cárie.

Descritores: Cárie Dentária; Criança; Reabilitação Bucal.

\section{Abstract}

Introduction: Severe childhood caries generates an economic, emotional and physical impact on children and their families, in addition to determining functional, aesthetic and behavioral alterations, which should be considered in the realization of a dental treatment Effective. Objective: The objective of this work was to conduct a clinical case report of aesthetic and functional rehabilitation of a 5-year-old male patient, affected by CSI, assisted in the discipline of Pediatric dental clinic of Universidade Federal of Amazonas. Clinical Case: On clinical and radiographic examination, carious lesions were observed in the dental elements 51, 52,61, 62 and 64, in addition to active white spot in the 74. The treatment proposed for this patient included orientation of adequate oral and dietary hygiene habits, topical fluoride applications in active carious lesions, necropulpectomy and restoration with light-cured composite resin in the 64 tooth, as well as in teeth 51, 52, 61 and 62, severely destroyed by caries disease, using acetate crowns. Conclusion: The treatment proved to be successful, being possible to perform in an outpatient setting, allowing to reestablish the masticatory function and the aesthetic of the smile, besides promoting health education through the awareness of the child/nucleus The importance of self-care in the elimination of etiological factors and in the control of caries disease.

Descriptors: Dental Caries; Child; Oral Rehabilitation.

\section{Resumen}

Introducción: La caries infantil severa tiene un impacto económico, emocional y físico en los niños y sus familias, además de determinar cambios funcionales, estéticos y de comportamiento, que deben considerarse en la efectividad de un tratamiento dental efectivo. Objetivo: El objetivo de este estudio fue informar un caso clínico de rehabilitación estética y funcional de un niño de 5 años afectado por CSI, que asistía a la Clínica Dental Infantil de la Universidad Federal de Amazonas. Reporte de Caso: el examen clínico y radiográfico reveló lesiones cariosas cavitadas en los elementos dentales 51,52,61,62 y 64, además de la mancha blanca activa 74. El tratamiento propuesto para este paciente incluyó orientación sobre hábitos de higiene oral y en la dieta, aplicaciones tópicas adecuadas de fluoruro en lesiones cariosas activas, necropulpectomía y restauración con resina compuesta fotopolimerizable en el diente 64, así como en los dientes 51,52,61 y 62 severamente destruidos por caries con coronas de acetato. Conclusión: El tratamiento demostró ser exitoso, siendo posible realizarlo de forma ambulatoria, lo que permitió restablecer la función de masticación y la estética de la sonrisa, además de promover la educación para la salud a través de la conciencia del núcleo del niño / familia sobre la importancia del autocuidado en eliminación de factores etiológicos y control de la enfermedad de caries.

Descriptores: Caries Dental; Niño; Rehabilitación Bucal.

\section{INTRODUÇÃO}

A cárie dentária, apesar da redução em sua prevalência na última década, ainda é um problema de saúde pública mundial que comumente afeta indivíduos de todas as idades, sendo considerada uma das doenças crônicas não transmissíveis mais prevalentes em crianças nos primeiros anos de vida ${ }^{1,2}$.

Diversos termos foram propostos ao longo dos anos para designar o padrão específico da cárie dentária em crianças pequenas. Entretanto, em 2005, a Academia Americana de Odontopediatria designou a Cárie Precoce da Infância (CPI) como a presença de um ou mais dentes decíduos cariados (cavitados ou não), perdidos (devido à cárie) ou restaurados em crianças até 71 meses de idade. A existência de qualquer sinal de lesão de cárie em superfície lisa em crianças menores de 3 anos de idade é indicativa de Cárie Severa da Infância (CSI). Em crianças de 3 a 5 anos de idade, a presença de pelo menos um dente anterior superior com cavidade, perdido ou restaurado por cárie, ou ainda, a presença de 4 ou mais superfícies cariadas, perdidas ou restauradas aos 3 anos, 5 ou mais superfícies aos 4 anos e 6 ou mais aos 5 anos de idade também caracterizam a CSI. Entretanto, há ainda crítica aos parâmetros atuais para o diagnóstico da CPI (idade da criança e envolvimento da dentição decídua), constituindo um desafio aos cirurgiões-dentistas a classificação de lesões de cárie em determinados $\operatorname{casos}^{3,4}$. 
Mais recentemente, a Declaração de Bangkok da Associação Internacional de Odontopediatria propôs a terminologia "Cárie na Primeira Infância" a fim de obter apoio em todo o mundo para uma definição baseada em evidências e uma compreensão em relação à etiologia, fatores de risco e intervenções para reduzir a doença, assim como estimular abordagens e políticas colaborativas para diminuir esta doença crônica 5 .

A ocorrência da CSI gera um impacto econômico, emocional e físico nas crianças e em suas famílias, afetando principalmente a alimentação e o sono, por desencadear um quadro de infecção, dor, dificuldade de mastigação, trauma psicológico e perda prematura de dentes ${ }^{2,4}$. Desta forma, determina um grande impacto negativo na qualidade de vida das crianças, com possível perda da autoestima e futuros danos à dentição permanente ${ }^{6}$.

O tratamento reabilitador para a CSI costuma ser um desafio para o odontopediatra, pois a pouca idade da criança geralmente implica em baixa colaboração e cooperação durante os procedimentos. Além disso, pequena quantidade de remanescente dental, menores valores de resistência de união do sistema adesivo ao dente e dificuldades inerentes à execução da técnica operatória tornam o tratamento reabilitador em crianças difícil, podendo resultar em insucesso ${ }^{7}$.

Apesar da diversidade de técnicas e materiais restauradores, os cirurgiões-dentistas devem estar sempre atentos em atender o melhor interesse de seus pacientes/núcleo familiar, lançando mão de técnicas e materiais com bom desempenho cientificamente comprovado ao longo do tempo. A restauração direta em resina composta com coroas de acetato, associada ou não ao tratamento endodôntico e ao uso de pino de fibra de vidro, tem se mostrado uma ótima alternativa na reabilitação da forma e da função dos dentes anteriores afetados, possibilitando a ressocialização da criança ${ }^{4,6,8,9}$.

Com base na literatura existente, esse relato torna-se significativamente relevante para a área odontológica, vindo agregar conhecimento cientifico sobre a eficácia da técnica restauradora usando coroas de acetato, utilizada como alternativa no tratamento da cárie dentária em crianças. Assim, o objetivo do presente estudo será realizar um relato de caso de uma reabilitação estética e funcional em um paciente acometido por CSI.

\section{CASO CLÍNICO}

Paciente do sexo masculino, com 5 anos de idade, compareceu à Clínica Odontológica Infantil II da Faculdade de Odontologia da Universidade Federal do Amazonas (Manaus-AM), acompanhado de sua mãe, para avaliação clínica de sua saúde bucal, com a queixa principal de que há mais de um ano seus dentes anteriores estavam escurecidos e bastante destruídos, e que, por isso, vinha sofrendo bullying na escola. Durante a anamnese, constatou-se que o paciente tinha ausência de doenças sistêmicas ou hipersensibilidade a medicamentos, apresentando boa saúde geral. E quando questionada sobre os hábitos alimentares da criança, a mãe respondeu que o mesmo consumia alimentos açucarados todos os dias e que fez uso de mamadeira noturna até os 3 anos. Ao exames clínico e radiográfico, foram constatadas extensas destruições coronárias dos elementos 52,51 , 61 e 62, com lesões de cárie ativas e sem envolvimento pulpar (Figuras 1 e 2).

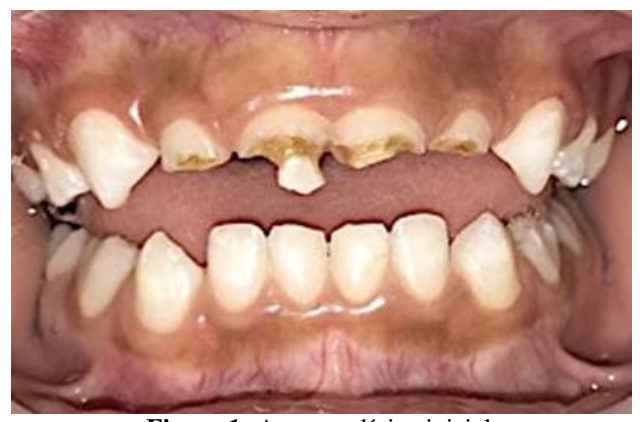

Figura 1: Aspecto clínico inicial.

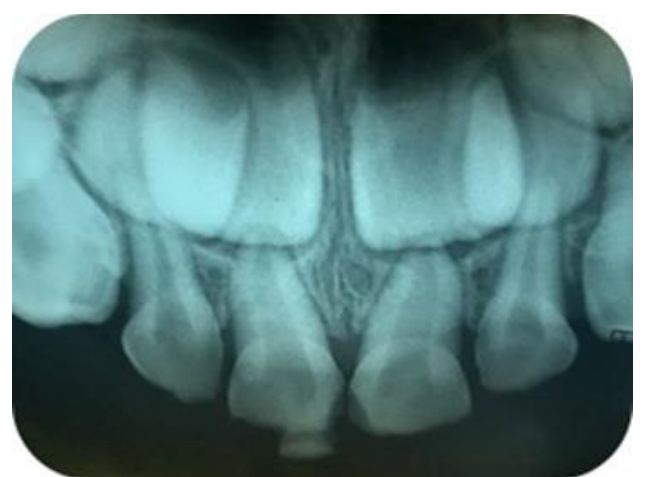

Figura 2: Aspecto radiográfico dos elementos dentários 52,51,61 e 62.

Também foi encontrada uma lesão de mancha branca ativa na face oclusal do elemento $74 \mathrm{e}$ uma lesão cariosa ativa com cavitação profunda na face oclusal do elemento 64, que apresentava uma fístula (Figura 3). Com o diagnóstico de Cárie Severa da Infância, foi realizado o planejamento do tratamento odontológico do paciente.

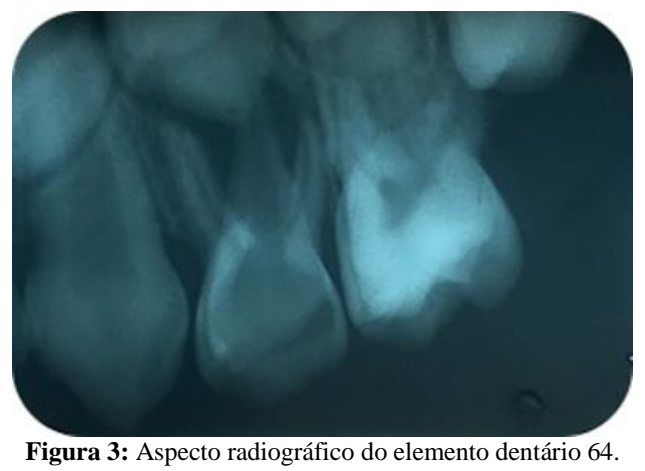

Inicialmente, o paciente e a responsável foram orientados e motivados quanto à prática dos hábitos de higiene bucal (orientações acerca da necessidade da realização da escovação pelo responsável, sua frequência, e do uso de dentifrício 
fluoretado, bem como de fio dental) e dietéticos (consumo racional de sacarose) adequados, sendo posteriormente realizada a profilaxia com pasta profilática e a primeira aplicação tópica de flúor nas lesões com atividade de cárie.

Na segunda consulta, o paciente encontravase bastante ansioso e agitado, não sendo possível dar início ao tratamento endodôntico do elemento 64, sendo realizada apenas a profilaxia e a $2^{\mathrm{a}}$ sessão de aplicação tópica de flúor, usando-se a técnica de manejo comportamental "modelagem", para que o mesmo observasse o tratamento realizado em outros pacientes infantis cooperadores.

$\mathrm{Na}$ terceira consulta, após o condicionamento do paciente, foi realizada a primeira sessão do tratamento endodôntico do elemento 64. Para isso realizou-se anestesia tópica com benzocaína 20\%, seguida de bloqueio de campo do nervo alveolar superior médio com articaína 4\% + epinefrina 1:100. Após, procedeu-se o isolamento absoluto, remoção do tecido cariado com cureta de dentina número 11,5 , cirurgia de acesso com uma broca esférica diamantada 1012 e odontometria. A seguir, realizouse o preparo químico-mecânico com limas rotatórias, irrigação dos condutos com solução de digluconato de clorexidina $2 \%$ e aspiração. Devido à agitação do paciente, optou-se pela colocação de medicação intracanal e selamento provisório duplo com uma camada de um material de preenchimento temporário e uma camada de cimento de ionômero de vidro restaurador autopolimerizável, realizando-se, em seguida, a profilaxia e a terceira aplicação tópica de flúor no paciente.

N Na quarta consulta, após a remoção do selamento provisório e da medicação, procedeu-se a obturação dos canais radiculares com pasta de hidróxido de cálcio, associado ao iodofórmio, e realizou-se a radiografia periapical para comprovar a obturação satisfatória dos canais radiculares (Figura 4). Em seguida, executou-se a restauração com resina composta fotopolimerizável do referido elemento dentário (Figura 5), seguida da $4^{\mathrm{a}}$ aplicação tópica de flúor.

$\mathrm{Na}$ consulta seguinte, selecionou-se e adaptou-se as coroas de acetato a serem usadas nas restaurações dos dentes anteriores superiores decíduos (Figura 6). Posteriormente, realizou-se o preparo cavitário dos elementos 52, 51, 61 e $62 \mathrm{com}$ broca carbide esférica e o preenchimento das coroas com resina composta, sendo fotopolimerizadas por vestibular, palatina e incisal. Posteriormente, foram retiradas com auxílio de uma sonda exploradora, checada a oclusão e procedeu-se a remoção dos excessos das restaurações com brocas diamantadas (Figura 7). Após um ano de proservação, observamos a ausência da doença cárie, bem como da sintomatologia que a paciente se queixava, resultando no sucesso do tratamento (Figura 8).

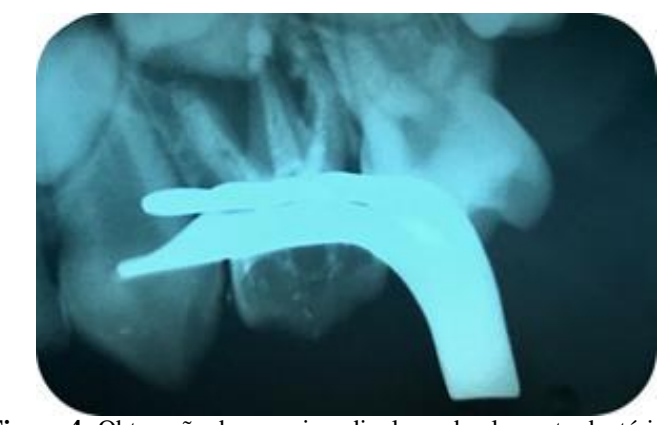

Figura 4: Obturação dos canais radiculares do elemento dentário 64.

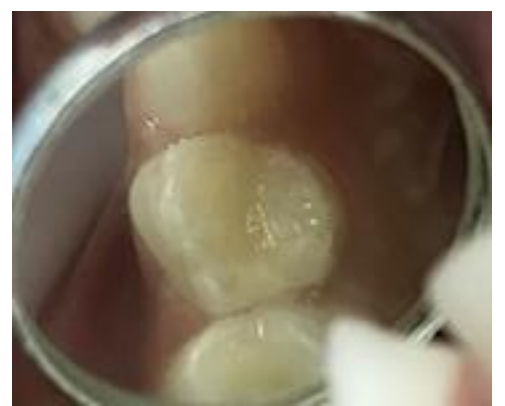

Figura 5: Restauração de resina composta no elemento dentário 64.

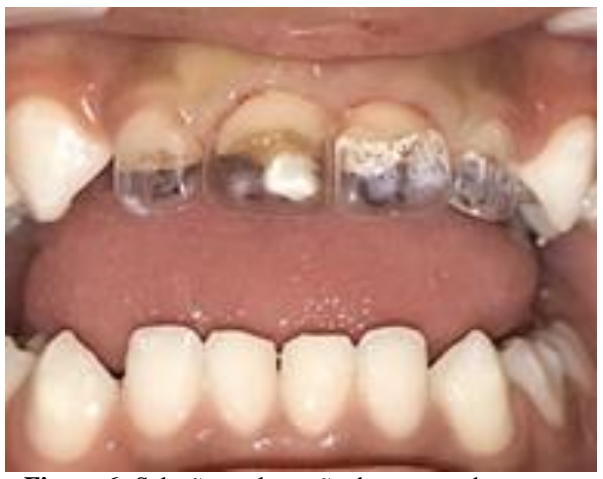

Figura 6: Seleção e adaptação das coroas de acetato.

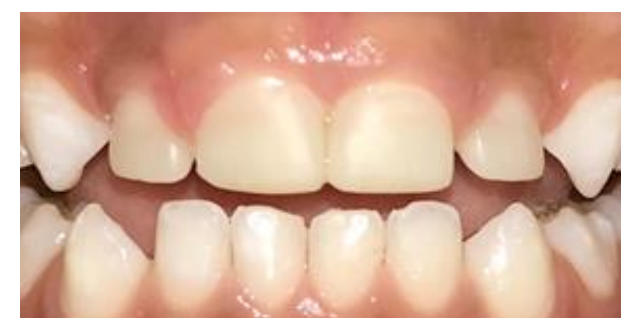

Figura 7: Aspecto clínico final das restaurações de resina composta dos elementos dentários 52,51,61 e 62 .

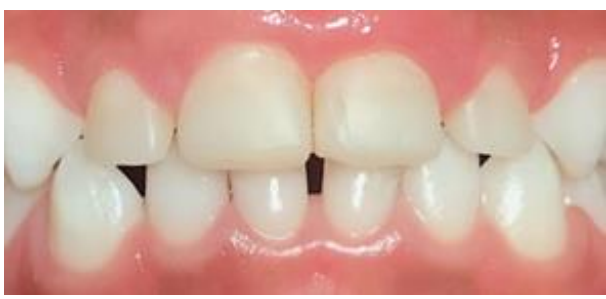

Figura 8: Proservação após um ano

DISCUSSÃO

Vários termos utilizados no passado para descrever a cárie na infância caíram em desuso na medida em que a multifatoriedade da doença cárie foi sendo esclarecida, além do que traziam uma ideia errônea e incompleta da causa da doença, conduzindo a uma conduta de tratamento que não alcançava o completo sucesso, uma vez que muitos outros fatores eram ignorados. O termo "Cárie Precoce da Infância" 
passou, então, a abranger de forma mais adequada a doença cárie que acometeria crianças abaixo dos 6 anos de idade; entretanto, esse termo vem sendo bastante discutido em razão da dificuldade de classificação das lesões de cárie quando o diagnóstico for tardio, embora a doença tenha se iniciado antes de 72 meses, ou ainda, quando da presença da doença em primeiros molares permanentes erupcionados precocemente ${ }^{3,4}$. Desta forma, a terminologia "Cárie na Primeira Infância" foi proposta para amenizar tal problema, mas ainda carece de aceitação na literatura científica $^{5}$. O paciente do presente caso clínico apresentava uma modalidade agressiva da CPI que recebe a nomenclatura de "Cárie Severa da Infância" (CSI), a qual pode se manifestar em crianças entre 3 e 5 anos de idade, da seguinte maneira: a) um ou mais dentes cavitados, perdidos (devido à cárie) ou com restauração em superfície lisa de dente anterior superior; b) cariado, perdido ou restaurado em 4 ou mais faces aos 3 anos, 5 ou mais faces aos 4 anos ou 6 ou mais faces aos 5 anos $^{4}$.

A CSI, como outras formas de cárie, é considerada uma doença dinâmica multifatorial, determinada pelo consumo de açúcar e mediada por biofilme que resulta no desequilíbrio entre os processos de des e remineralização dos tecidos duros dentários. A cárie dentária é determinada por fatores biológicos, comportamentais e psicossociais relacionados ao meio do indivíduo. Compartilha fatores de risco comuns a outras doenças não transmissíveis (DNT) associadas ao consumo excessivo de açúcar, como doença cardiovascular, diabetes e obesidade. O consumo excessivo de açúcar determina produção prolongada de ácidos a partir de bactérias que se aderem ao dente e uma mudança na composição da microbiota oral e $\mathrm{pH}$ do biofilme. Se mantido, as estruturas do dente são desmineralizadas $^{10,11}$. A presença de defeitos de desenvolvimento do esmalte, a baixa renda familiar e a baixa escolaridade também podem estar associadas à $\mathrm{CPI}^{1,4,12}$. Concordando com o exposto na literatura pesquisada, o menor em questão possuía hábitos dietéticos inadequados, pois consumia alimentos açucarados todos os dias e tinha o hábito de mamadeira noturna até os 3 anos, além dos pais apresentarem baixa renda e baixa escolaridade.

Por ser determinada por fatores comportamentais, a orientação quanto aos hábitos dietéticos e de higiene bucal é significativa no sucesso do tratamento da cárie dentária e, além disso, é de extrema importância na manutenção do equilíbrio e da saúde geral da criança ${ }^{1,2}$. No caso clínico descrito, a orientação quanto aos hábitos dietéticos, bem como aos de higiene bucal adequados foram repassados à mãe e reforçados ao longo de todas as consultas, medidas estas também descritas em outro estudo ${ }^{6}$.

A prevenção e o tratamento de CPI podem ser estruturados em três fases: a) Prevenção primária, a qual objetiva melhorar o conhecimento de pais/cuidadores e trabalhadores em saúde, limitando o consumo infantil de açúcares livres em bebidas e comidas, além de propiciar a exposição diária ao flúor; b) Prevenção secundária, que consiste no controle efetivo de lesões iniciais antes da cavitação (aplicações de verniz fluoretado e de selantes de fissura); c) Prevenção terciária, que inclui a paralisação de lesões cavitadas e o tratamento operatório preservando a estrutura dentária ${ }^{5}$. No presente caso clínico, o manejo apropriado da CPI a partir da informação dos pais e da aplicação de verniz fluoretado nas lesões ativas de cárie (fases primária e secundária da prevenção/tratamento) foram importantes para reduzir o impacto desta doença prevenível, assim como relatado por Neves et al. ${ }^{8}$.

A CPI e a CSI impacta negativamente na qualidade de vida de crianças e suas famílias e representa um impacto desnecessário para a sociedade, causando dores e desconforto, dificuldades na alimentação, no sono, no aprendizado, faltas escolares, problemas de autoestima e de autoconfiança ${ }^{5,6,8,11}$. Todas essas consequências podem prejudicar o desenvolvimento físico e psicológico da criança, além de refletir sobre o núcleo familiar levando, inclusive, a gastos inesperados inerentes ao tratamento, faltas em dias de trabalho para cuidar da criança, dentre outros. A criança descrita no caso clínico em questão sofria bullying na escola, tendo dificuldade na interação social e excessiva timidez, além de apresentar dores e dificuldades durante a mastigação, da mesma forma que o paciente infantil atendido por Neves et al. ${ }^{8}$, cuja mãe relatou dificuldade no relacionamento social de seu filho com os colegas por apresentar os "dentes estragados".

A reabilitação bucal em crianças com CSI é um desafio para os Odontopediatras e uma correta abordagem multidisciplinar determina o sucesso do tratamento $^{12}$. O sucesso do tratamento restaurador convencional depende de alguns fatores como a técnica, os materiais usados e a cooperação da criança tanto durante o procedimento quanto após, ao aderir, junto ao núcleo familiar, a uma adequada dieta e a uma boa higiene bucal, havendo necessidade de estudos adicionais sobre novas alternativas de tratamento e do cuidado do profissional em escolher o recurso terapêutico mais apropriado para cada caso $^{6}$. De acordo com Lourenço Neto et al. ${ }^{7}$, o Odontopediatra deve optar pelo tratamento que alcance o resultado estético e funcional, mas a escolha deve ser individualizada e estar calcada na aceitabilidade de cada paciente e de seu núcleo familiar.

Além das dificuldades técnicas relacionadas aos tratamentos, somam-se a isso, os problemas inerentes ao controle da conduta de um paciente 
muito jovem, o que traz a necessidade de um maior conhecimento de técnicas de manejo comportamental e uma habilidade no trato odontológico por parte do profissional, visando ao bem-estar de ambos nestas circunstâncias $^{6,7}$. O paciente infantil deste caso clínico, nas primeiras consultas, se encontrava bastante ansioso e agitado, chorava bastante, tendo sido condicionado ao longo do tratamento por meio de técnicas de manejo comportamental, como a modelagem e o dizer-mostrar-fazer, chegando ao final do tratamento odontológico reabilitador com um comportamento bastante cooperador.

Os cirurgiões-dentistas devem estar sempre atentos em atender o melhor interesse de seus pacientes, lançando mão de técnicas e materiais com bom desempenho cientificamente comprovado ao longo do tempo. A restauração direta em resina composta com coroas de acetato, associada ou não ao tratamento endodôntico e ao uso de pino de fibra de vidro, tem se mostrado uma ótima alternativa, simples e rápida, na reabilitação da forma e da função dos dentes anteriores afetados (fase terciária da prevenção/tratamento da CPI), possibilitando a ressocialização da criança ${ }^{5,6,9}$.

A reabilitação bucal é essencial para melhoria dos aspectos psicossociais da criança e para o desenvolvimento de funções como fonação, deglutição e mastigação, além de devolver qualidade de vida ao paciente e satisfação à sua família, Corroborando com o exposto, o menor do presente caso, na consulta odontológica da reabilitação dos dentes anteriores superiores, demonstrou uma alegria intensa, mostrando o sorriso a todos os presentes na clínica. Observou-se, desta forma, mudança extremamente positiva em relação à percepção da criança quanto à reabilitação de seus dentes, a qual se mostrou muito satisfeita com o resultado obtido e extremamente motivada e empenhada, junto ao seu núcleo familiar, com os cuidados relativos à higiene bucal. Foi também relatada pela mãe uma melhora na qualidade e na quantidade da alimentação da criança, além de não haver mais a queixa de dores ao mastigar.

O tratamento proposto para o caso clínico descrito neste trabalho incluiu procedimentos restauradores, endodônticos e periodontais, visando à manutenção dos dentes decíduos até a época geneticamente determinada para sua esfoliação fisiológica $^{2,4}$, além da instituição de medidas educativas de higiene bucal, orientação de dieta e fluorterapia, assemelhando-se aos propostos por Coclete et al. ${ }^{6}$, Laranjo et al. ${ }^{11}$ e Souza et al. ${ }^{9}$, onde a escolha de técnicas restauradoras adesivas diretas, opção de tratamento executada em ambiente ambulatorial, proporciona custo relativamente menor por não envolver etapa laboratorial. O uso de coroas de acetato em restaurações de resina composta é uma alternativa útil na reabilitação de dentes decíduos severamente destruídos, fraturados ou com defeitos de desenvolvimento do esmalte, principalmente em crianças pequenas, proporcionando às restaurações durabilidade, bom resultado estético, menor tempo clínico e baixo custo, quando comparadas às coroas de zircônia ${ }^{9,13}$, possibilitando o restabelecimento da estética e função dos elementos dentários, bem como a autoestima dos pacientes.

As orientações e esclarecimentos prestados ao núcleo familiar da criança acometida pela CSI são de extrema importância para o sucesso do tratamento e manutenção da saúde bucal. Muitas vezes, o Odontopediatra vai além dos procedimentos técnicos e participa da reabilitação bucal da criança, auxiliando na mudança comportamental da criança e de seu núcleo familiar, além de muitas vezes agir como apoio psicológico à família. Agindo desta forma, o profissional está aplicando amplamente o conceito de educação para a saúde que se constitui num dos caminhos para a conscientização e prevenção quanto à doença na infância e na vida adulta.

\section{CONCLUSÃO}

Conclui-se que é possível reabilitar uma criança, mesmo que de baixa idade, pois muitos profissionais acabam postergando a realização do tratamento odontológico definitivo no aguardo da colaboração e cooperação da mesma e que o uso de coroas de acetato nas restaurações de resina composta é uma alternativa útil para a reabilitação bucal de crianças pequenas, por ser uma técnica de simples execução, baixo custo, de menor tempo clínico, capaz de restabelecer a estética e a autoestima dos pacientes. Torna-se imprescindível na obtenção da saúde geral e bucal da criança que o Odontopediatra, além da realização dos procedimentos técnicos, forneça orientações e promova a educação para a saúde através da conscientização da criança e de seu núcleo familiar acerca da importância do autocuidado na eliminação dos fatores etiológicos e no controle da doença cárie.

\section{REFERÊNCIAS}

1. Alazmah A. Early childhood caries: a review. J Contemp Dent Pract. 2017;18(8):1-6.

2. Percival T, Edwards J, Barclay S, Sa B, Majumder MAA. Early childhood caries in 3 to 5 year old children in Trinidad and Tobago. Dent J. 2019; 7(1):16.

3. Folayan M, Olatubosun S. Early childhood caries: a diagnostic enigma. Eur J Paediatr Dent. 2018; 19(2):88.

4. Silva MGB, Catão MHCV, Andrade FJP, Alencar CRB. Cárie precoce da infância: fatores de risco associados. Arch Health Invest. 2017;6(12):574-9.

5. Early Childhood Caries: IAPD Bangkok Declaration. Int J Paediatr Dent. 2019;29(3):384-86.

6. Coclete GEG, Nagata ME, Hosida TY, Báez- 
Quintero C, Cunha RF, Pessam JP. Cárie precoce da infância: reabilitação estética e funcional. Arch Health Invest. 2016;5(1):237.

7. Lourenço Neto N, Cardoso CB, Abdo RCC, Silva SMB. Oral rehabilitation in pediatric dentistry: a clinical case report. Rev Gaúch Odontol. 2016; 64(1):87-91.

8. Neves FAS, Brandão CF, Freitas LMA, Simões FXPC. Reabilitação estético-funcional em dentes decíduos: relato de um caso clínico. Rev. UNINGÁ. 2017;54(1):167-75.

9. Souza, MIAV, Cavalheiro JP, Bussaneli DG, Jeremias F, Zuanon ACC. Aesthetic A with strip crowns in pediatric dentistry: a case report. Rev CES Odont. 2018; 31(2):66-75.

10. Chen KJ, Gao SS, Duangthip D, Lo ECM, Chu $\mathrm{CH}$. Managing early childhood caries for young children in China. Healthcare. 2018;6(1):1-9.

11. Laranjo E, Baptista S, Norton AA, Macedo AP, Andrade C, Areias C. A cárie precoce da infância: uma atualização. Rev Port Med Geral Fam.2017; 33(6):426-29.

12. Kirthiga M, Murugan M, Saikia A., Kirubakaran R. Risk factors for early childhood caries: a systematic review and meta-analysis of case control and cohort studies. Pediatr Dent. 2019; 41(2):95-114.

13. Aiem E, Smail-Faugeron V, Muller-Bolla M. Aesthetic preformed paediatric crowns: systematic review. Int J Paediatr Dent.2017;27(4):273-82.

\section{CONFLITO DE INTERESSES}

Os autores declaram não haver conflitos de interesse.

\section{AUTOR PARA CORRESPONDÊNCIA}

Simone Assayag Hanan

Faculdade de Odontologia,

Universidade Federal do Amazonas (UFAM)

Av. Waldemar Pedrosa, 1539,

69033-760. Manaus, AM, Brasil

+55(92)3305-4905.

E-mail: simonehanan@yahoo.com.br

Submetido em 15/01/2020

Aceito em 13/06/2020 